\title{
Replication of Brucella abortus and Brucella melitensis in fibroblasts does not require Atg5-dependent macroautophagy
}

\author{
Isabelle Hamer ${ }^{1 *}$, Emeline Goffin ${ }^{1,3}$, Xavier De Bolle ${ }^{2}$, Jean-Jacques Letesson ${ }^{2}$ and Michel Jadot ${ }^{1}$
}

\begin{abstract}
Background: Several intracellular bacterial pathogens have evolved subtle strategies to subvert vesicular trafficking pathways of their host cells to avoid killing and to replicate inside the cells. Brucellae are Gram-negative facultative intracellular bacteria that are responsible for brucellosis, a worldwide extended chronic zoonosis. Following invasion, Brucella abortus is found in a vacuole that interacts first with various endosomal compartments and then with endoplasmic reticulum sub-compartments. Brucella establishes its replication niche in ER-derived vesicles. In the past, it has been proposed that B. abortus passed through the macroautophagy pathway before reaching its niche of replication. However, recent experiments provided evidence that the classical macroautophagy pathway was not involved in the intracellular trafficking and the replication of B. abortus in bone marrow-derived macrophages and in HeLa cells. In contrast, another study showed that macroautophagy favoured the survival and the replication of Brucella melitensis in infected RAW264.7 macrophages. This raises the possibility that B. abortus and B. melitensis followed different intracellular pathways before replicating. In the present work, we have addressed this issue by comparing the replication rate of B. abortus and B. melitensis in embryonic fibroblasts derived from wild-type and Atg $5^{-/-}$mice, Atg5 being a core component of the canonical macroautophagic pathway.
\end{abstract}

Results: Our results indicate that both B. abortus S2308 and B. melitensis $16 \mathrm{M}$ strains are able to invade and replicate in Atg5-deficient fibroblasts, suggesting that the canonical Atg5-dependent macroautophagic pathway is dispensable for Brucella replication. The number of viable bacteria was even slightly higher in $\mathrm{Atg}^{5^{-1-}}$ fibroblasts than in wild-type fibroblasts. This increase could be due to a more efficient uptake or to a better survival rate of bacteria before the beginning of the replication in Atg5-deficient cells as compared to wild-type cells. Moreover, our data show that the infection with $B$. abortus or with $B$. melitensis does not stimulate neither the conversion of LC3-I to LC3-II nor the membrane recruitment of LC3 onto the BCV.

Conclusion: Our study suggests that like Brucella abortus, Brucella melitensis does not subvert the canonical macroautophagy to reach its replicative niche or to stimulate its replication.

Keywords: Brucella abortus, Brucella melitensis, Intracellular trafficking, Replication, Macroautophagy, Atg5

\section{Background}

Many intracellular bacteria have developed strategies to hijack the intracellular trafficking machinery of the host cell in order to escape lysosomal degradation ensuring their survival and their replication [1]. For example, Mycobacterium tuberculosis blocks the maturation of phagosomes into the degradative phagolysosomes by producing

\footnotetext{
* Correspondence: isabelle.hamer@unamur.be

${ }^{1}$ Research Unit in Molecular Physiology (URPhyM), NAmur Research Institute for LIfe Sciences (NARILIS), University of Namur, Namur, Belgium

Full list of author information is available at the end of the article
}

lipids that mimic the phosphoinositides and inhibit the fusion between phagosomes and lysosomes [2]. Some bacteria, including Coxiella burnetii, Legionella pneumophila and Staphylococcus aureus can survive and replicate for some time in autophagosome-like vacuoles by delaying [3,4] or by blocking [5] their maturation into autophagolysosomes.

After its uptake by HeLa cells, Brucella abortus is recovered in a vacuole $(\mathrm{BCV})$ that transiently interacts with early and late endosomes and perhaps lysosomes, successively acquiring markers of endosomal compartments such 
as EEA1 (Early Endosome Antigen 1), Rab5, Rab7 and LAMP-1 (Lysosomal-associated membrane protein 1) [6]. During these different steps of maturation, the BCV becomes acidic allowing the expression of genes encoding the VirB type IV secretion system (T4SS) [6]. Brucella avoids lysosomal degradation by blocking the phagosomelysosome fusion probably by a mechanism dependent on lipid rafts and perhaps on cyclic $3-1,2$-glucans [7-9]. Afterwards, the BCV interacts in a sustained way with subdomains of the endoplasmic reticulum, called ERES (endoplasmic reticulum exit sites) and at around $12 \mathrm{~h}$ p.i., Brucella abortus starts to replicate in ER-derived vesicles labelled with ER specific markers, such as sec61ß and calnexin $[6,10,11]$. Later on, from 48 h p.i., Starr et al. [12] demonstrated that these replicative $\mathrm{BCV}(\mathrm{rBCV})$ could be converted into LAMP-1 and Rab7-positive compartments (called autophagic BCV or aBCV) that would be involved in the completion of the intracellular Brucella lifecycle and could promote its cell-to-cell spreading [12].

Earlier studies had already revealed that some bacteria resided in autophagosome-like vacuoles characterized by multilamellar membranes after $24 \mathrm{~h}$ of infection and that Brucella replication was increased when macroautophagy was activated by serum starvation, suggesting that $B$. abortus transits through the autophagic pathway before reaching its replicative compartment $[11,13]$. Since then, many proteins implicated in the regulation of macroautophagy (Atg proteins) have been discovered [14,15]. The initiation of autophagosome formation requires the ULK complex and the class III phosphatidylinositol 3-P kinase (PI3K) complex. The nucleation of the isolation membrane requires the recruitment of additional Atg proteins and autophagy-specific PtdIns(3)P effectors [14,15]. The expansion of the isolation membrane relies on two ubiquitylation-like reactions. The first one drives the conjugation of Atg12 to Atg5 in the presence of Atg7 and Atg10. Atg5-Atg12 conjugates are non-covalently associated to Atg16L (Atg16-like) forming multimeric complexes of approximately $800 \mathrm{kDa}$ [16-18]. The second reaction conjugates the cytosolic soluble LC3-I (microtubule-associated protein 1 light chain 3) to a phosphatidylethanolamine (PE) in the presence of Atg4, Atg3 and Atg7 producing the membrane-associated LC3-II form [19-21]. The Atg5-Atg12 conjugates are essential for the maturation of the isolation membrane into autophagosome and targeting of LC3 to the membrane [18].

Recently, using epithelial cells and macrophages deficient in one of the regulatory proteins of the conventional macroautophagic pathway, Starr et al. [12] have found that core proteins of this canonical macroautophagy machinery such as ULK-1, Beclin1, Atg5, Atg7, LC3B were not necessary for the intracellular trafficking of $B$. abortus between the endocytic compartments and the ER-derived vesicles and for its replication [12]. Nevertheless, the conversion of $\mathrm{rBCV}$ to $\mathrm{aBCV}$ at a later stage of infection, i.e. $48 \mathrm{~h}$ and 72 h p.i., seems to be dependent on ULK-1, Beclin1, Atg14L and hVps34 but independent on Atg5, Atg7, Atg16L1 and Atg4B [12]. On the other hand, Guo et al. [22] have observed that infection by $B$. melitensis induced macroautophagy that in turn favoured its replication in RAW264.7 macrophages [22]. This later study raises the possibility that in contrast to B. abortus, B. melitensis could subvert macroautophagy to replicate in host cells. In our present work, we addressed this issue using embryonic fibroblasts from wild-type and Atg5-knockout mice infected or not with B. abortus and B. melitensis.

\section{Results}

Relative abundance of LC3-I and LC3-II in infected mouse embryonic fibroblasts

As it has been shown that B. melitensis stimulated macroautophagy in macrophages to favour its replication [22], we sought to determine whether this also occurred in infected MEFs. First, we established clones stably transfected with GFP-LC3 to monitor the formation of autophagic vacuoles by fluorescence microscopy. As expected [19], in basal conditions, the fluorescent staining in GFP-LC3 expressing cells was faint and diffuse while under starvation conditions, it was more punctuate, due to the recruitment of LC3 onto autophagosomal membranes (Additional file 1). In contrast, when the same cells were infected with $B$. abortus or with B. melitensis, the GFP-LC3 staining remained diffuse and colocalisation between GFP-LC3 and Texas Red-labelled bacteria was only very occasionally detected. Then, we examined the relative abundance of LC3-I and LC3-II by Western blotting. Preliminary experiments showed that in WT MEFs, LC3-II was detected even in basal conditions (Figure 1A). After $2 \mathrm{~h}$ of starvation in EBSS, the abundance of both LC3-I and LC3-II decreased, probably due to an acceleration of the autophagic flow since LC3-II is degraded when autophagosomes fuse with lysosomes. In contrast, the LC3-II/LC3-I ratio increased in the presence of bafilomycin, a vacuolar $\mathrm{H}^{+}$-ATPase inhibitor known to block autophagosome/lysosome fusion. As expected, in Atg $5^{-/}$MEFs, LC3-II was never detected whatever the cell culture conditions because the presence of Atg5 is absolutely required for the LC3 recruitment onto autophagosome membrane [19]. In WT MEFs infected with $B$. abortus or with $B$. melitensis, the relative abundance of LC3-I and LC3-II at 18 h p.i. did not change when compared to non-infected MEFs (Figure 1B).

\section{Replication of $B$. abortus- and B. melitensis-mCherry in Atg $5^{-1-}$ fibroblasts}

We studied the contribution of the macroautophagic pathway on the replication of Brucellae using Atg5-deficient MEFs. First, we infected cells with B. abortus-mCherry (Figure 2A) or with B. melitensis-mCherry (Figure 2B) for 


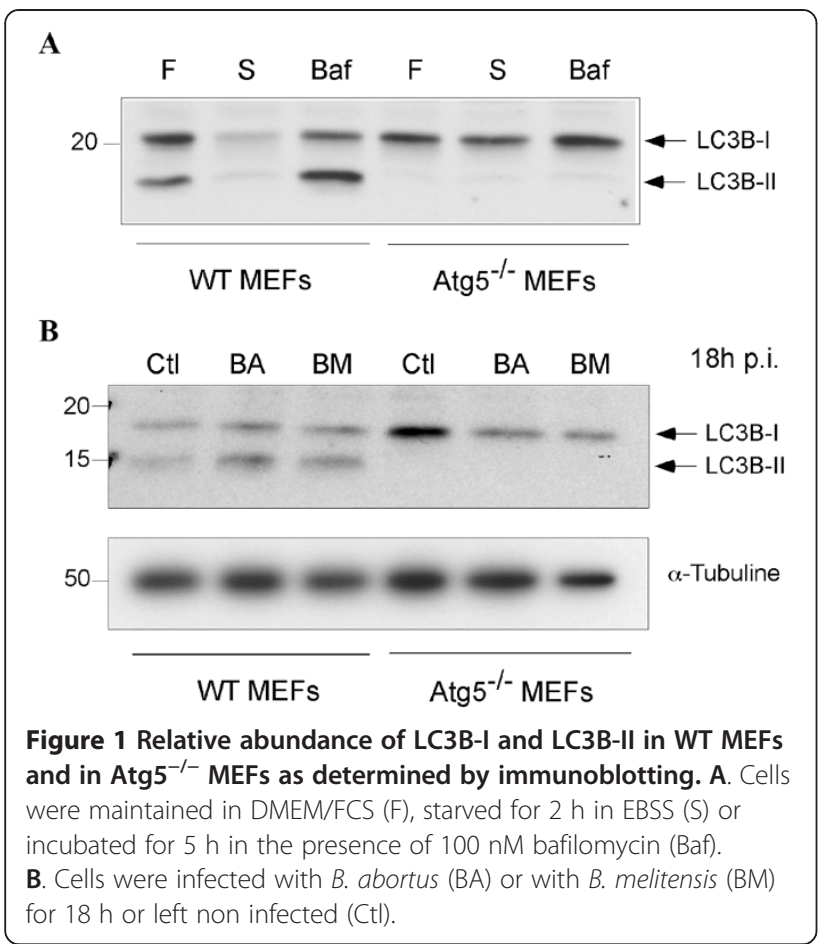

$1 \mathrm{~h}$ at a multiplicity of infection (MOI) of 300 . After inoculation, the medium was removed and replaced by a medium containing gentamicin to kill extracellular bacteria. As it can be seen on micrographs taken after increasing times postinfection, B. abortus-mCherry is able to enter, survive and replicate in MEFs, even in Atg5deficient MEFs. In both cell lines, at $6 \mathrm{~h} \mathrm{p.i,} \mathrm{there} \mathrm{are} \mathrm{only}$ a few bacteria per infected cell but this number massively increases between 12 and $18 \mathrm{~h}$ p.i. and at $24 \mathrm{~h}$ p.i., the bacteria are so abundant that it is difficult to enumerate them. B. melitensis-mCherry is also able to replicate in both WT MEFs and $\operatorname{Atg} 5^{-1-}$ MEFs. However, it is clear that the number of bacteria per infected cell at $24 \mathrm{~h}$ p.i. is lower compared to $B$. abortus-mCherry. Statistical analysis of these observations revealed that there is no significant difference in the number of $B$. abortus-mCherry per infected cell between the Atg5-deficient MEFs and the WT MEFs whatever the time postinfection (Figure 3A). In contrast, the number of $B$. melitensis-mCherry per infected cell significantly increased in Atg5 ${ }^{-/-}$MEFs when compared to WT MEFs at 9 h, $18 \mathrm{~h}$ and $24 \mathrm{~h}$ p.i. (Figure 3B). These data demonstrate that both Brucella strains can survive and replicate when the conventional Atg5-dependent macroautophagic pathway is impaired. Atg5-deficient cells seem to be even more permissive for $B$. melitensis replication than WT MEFs.

\section{Counting of viable bacteria in $\operatorname{Atg} 5^{-/-}$fibroblasts}

The counting of CFUs in the gentamicin survival assay represents a common way to investigate the survival and

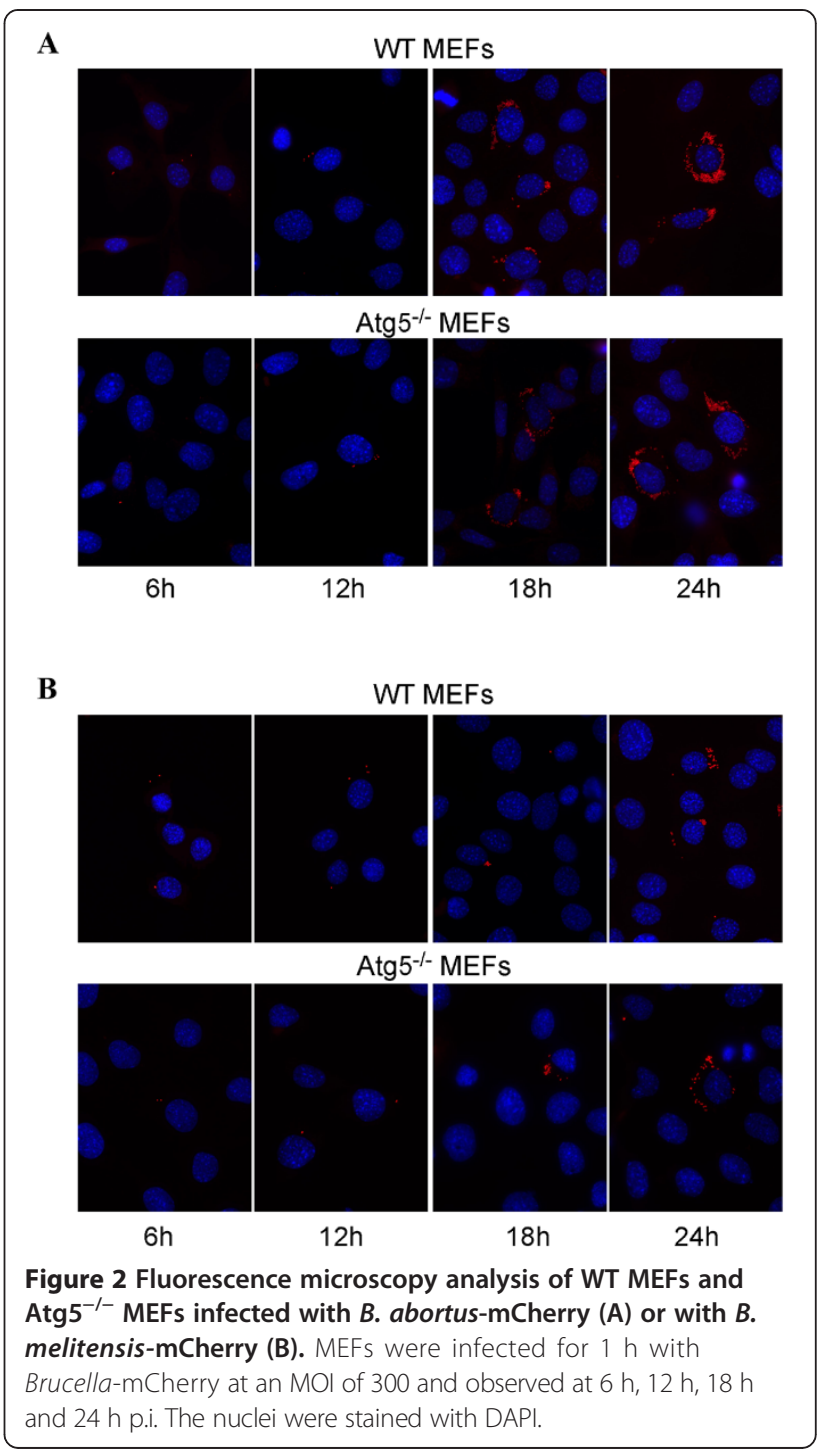

the replication of bacteria in host cells. In agreement with our morphological observations, we noticed that $B$. abortus grew at an exponential rate as a function of time postinfection both in WT and Atg5 $5^{-1-}$ MEFs (Figure 4A). There was even a slight increase in the $\log \mathrm{CFU}$ in Atg $5^{-1-}$ MEFs as compared to WT MEFs. A Student's t-test on each time point indicated that the difference between the WT and Atg $5^{-1-}$ MEFs was significant only at $12 \mathrm{~h}$ p.i. Nevertheless, a two-way ANOVA statistical analysis on all time points combined revealed that there was a highly significant increase in the $\log \mathrm{CFU}$ in $\mathrm{Atg} 5^{-1-}$ MEFs when compared to WT MEFs $(p<0.001)$. The same observation was made with $B$. melitensis (Figure 4B). This global increase could result from a more efficient uptake of bacteria rather than from a higher replication rate in $\mathrm{Atg} 5^{-/-}$ MEFs compared to WT MEFs. Alternatively, this increase in $\log$ CFU could be linked to a lower bactericidal capacity 


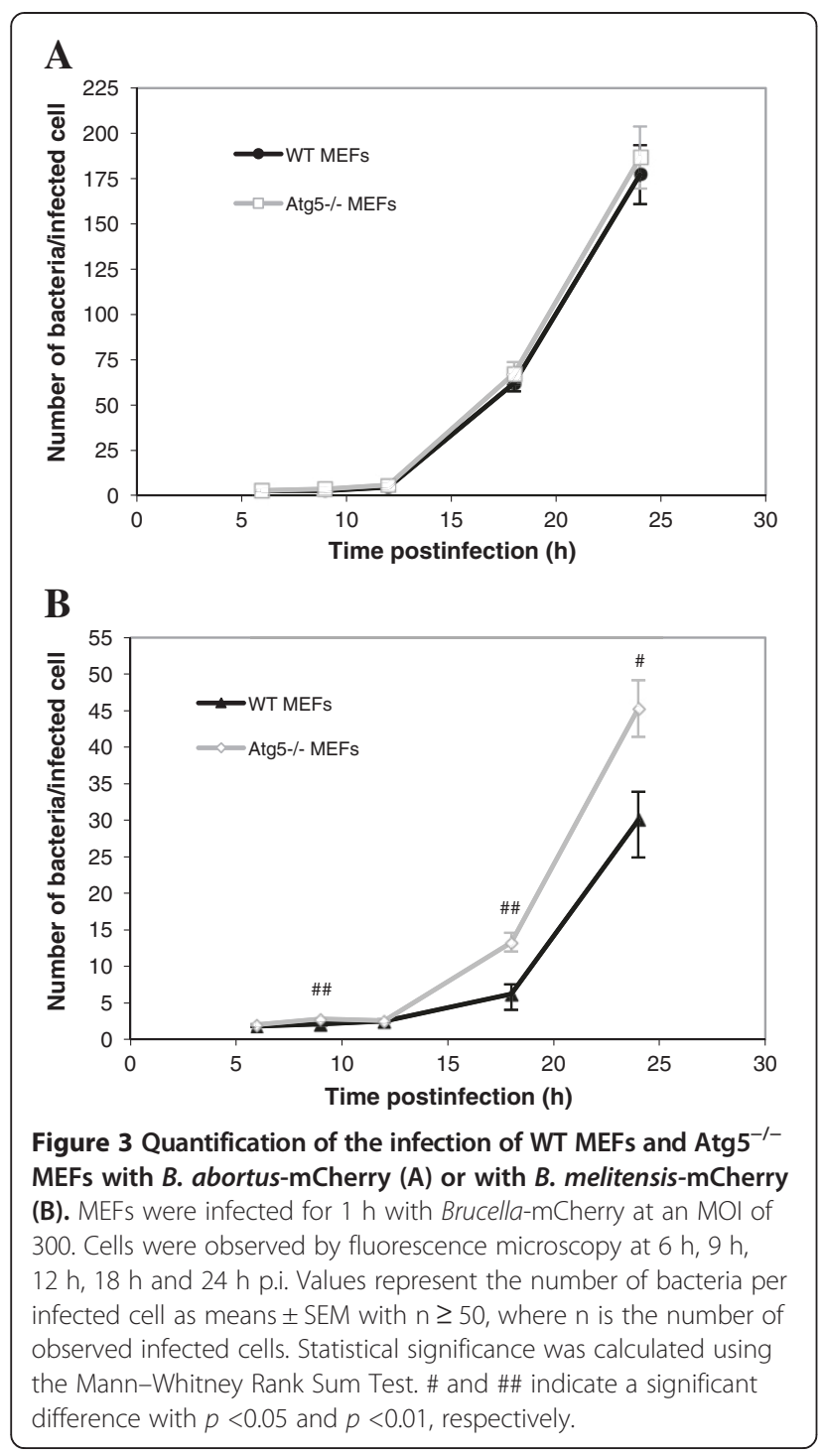

of Atg5-deficient cells compared to WT cells at early stages of infection.

\section{Intracellular replication of $B$. abortus and $B$. melitensis in the presence of 3-methyladenine}

Previous studies have shown that incubation of cells in the presence of 3-methyladenine (3MA), an inhibitor of class III PI3K often used to block macroautophagy [23], impaired the replication of B. abortus [13] and B. melitensis [22] in HeLa cells and in RAW264.7 macrophages, respectively. These data are in contradiction with our results showing that both bacterial strains are able to replicate in Atg5-deficient MEFs. Therefore, we sought to determine the putative impact of 3MA on the replication of Brucellae in WT MEFs. First, we assessed the number of $B$. abortus-mCherry per infected cell in WT MEFs preincubated for $2 \mathrm{~h}$ in the presence or absence of $10 \mathrm{mM}$ 3MA. As shown in Figure 5A, this treatment had no

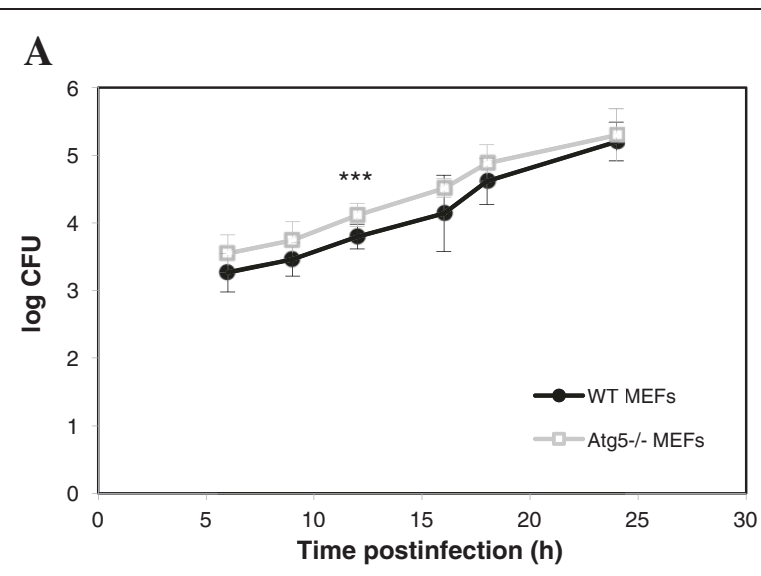

B

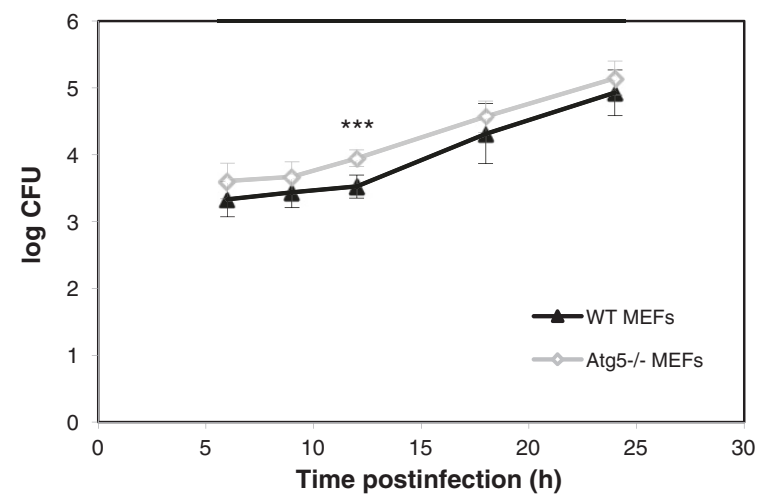

Figure 4 Intracellular growth of Brucella in WT and Atg5 $5^{-/-}$ MEFs. MEFs were infected for $1 \mathrm{~h}$ with B. abortus S2308 (A) or with B. melitensis 16M (B) at an MOI of 300. Log CFUs were obtained from cell lysates of infected WT MEFs and Atg $5^{-/-}$MEFs at the indicated time after infection. Results represent means \pm SD measured from at least three independent experiments made in triplicates. Statistical significance was calculated using the Holm-Sidak multiple comparisons test following a two-way ANOVA. $p<0.001$ for both B. abortus and B. melitensis. ${ }^{* *}$ indicates a highly significant difference using a Student's t-test.

significant impact on the number of bacteria per infected WT MEF. Similar results were obtained with WT MEFs infected with B. melitensis-mCherry (Figure $5 \mathrm{~B})$. However, in this case, we observed a significant decrease $(p<0.01)$ in the number of bacteria per infected cell but only at $24 \mathrm{~h}$ p.i. Next, we examined the impact of a pre-treatment with 3MA on Brucella replication in host cells using the gentamicin survival assay. Our results show that a pre-incubation of WT MEFs with 3MA does not impair the replication of both B. abortus and B. melitensis (Figure 6 A-B).

\section{Discussion}

After internalisation, B. abortus is found inside individual vacuoles that interact transiently with endosomes and perhaps lysosomes [6]. Then, Brucella evades the endocytic pathway and reaches its replicative niche, an ER-derived 


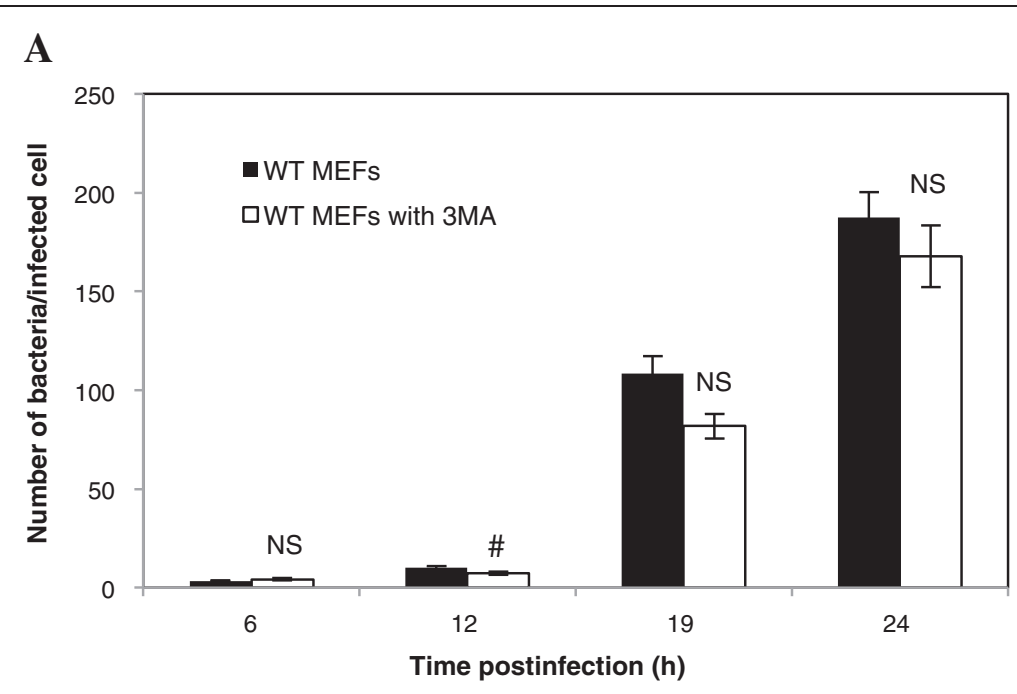

B

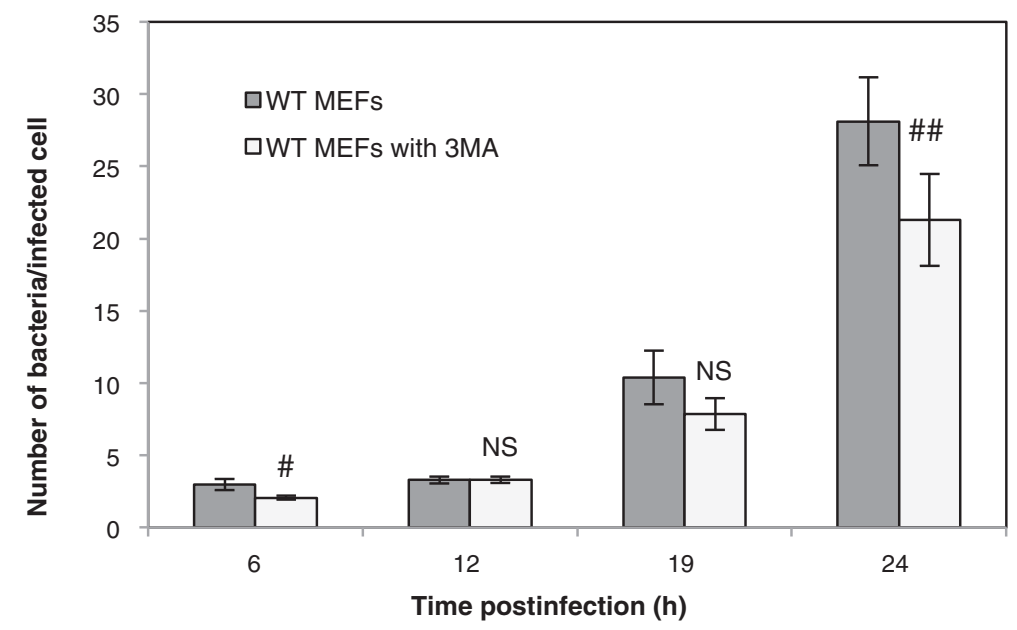

Figure 5 Impact of 3MA on the infection of WT MEFs with B. abortus-mCherry (A) or with B. melitensis-mCherry (B). The number of bacteria per infected cell was measured on at least 57 infected cells coming from two independent experiments. Values represent means \pm SEM. Statistical significance was calculated using the Mann-Whitney Rank Sum Test. \# and \#\# indicate a significant difference with $p<0.05$ and $p<0.01$, respectively. NS stands for non significant difference.

compartment, by a still unknown mechanism. It is also unclear whether Brucella transits through the autophagic pathway before its replication. Based on the appearance of B. abortus in multilamellar structures looking like autophagosomes and on the decrease of its replication rate after autophagy inhibition with 3MA, Pizarro-Cerda et al. [11] proposed that this bacterium passed through the autophagy pathway before reaching its niche of replication [13]. In agreement with this assumption, Guo et al. (2012) noticed that inoculation of macrophages with $B$. melitensis stimulated autophagy and that a pre-treatment with 3MA reduced its growth rate [22]. In contrast, using macrophages derived from KO mice or HeLa cells incubated in the presence of siRNA targeting the autophagic machinery, Starr et al. [12] showed that B. abortus does not use the conventional macroautophagic pathway either for its intracellular trafficking between the endocytic compartments and the ER derived-vesicles or for its replication [12]. In our study, we sought to compare the fate of $B$. abortus and B. melitensis in Atg5-deficient MEFs, i.e. in cells that are unable to set up the conventional pathway of macroautophagy even under starvation conditions.

Our results show that both Brucella strains are able to invade and replicate in Atg $5^{-1-}$ MEFs, indicating that Atg5 is dispensable for the intracellular survival and replication not only of $B$. abortus but also of $B$. melitensis. We observed even a slight but significant increase in the log CFU in $\operatorname{Atg} 5^{-1-}$ MEFs infected with B. abortus or with B. melitensis when compared to WT MEFs, all time points combined. The counting of fluorescent bacteria per infected 


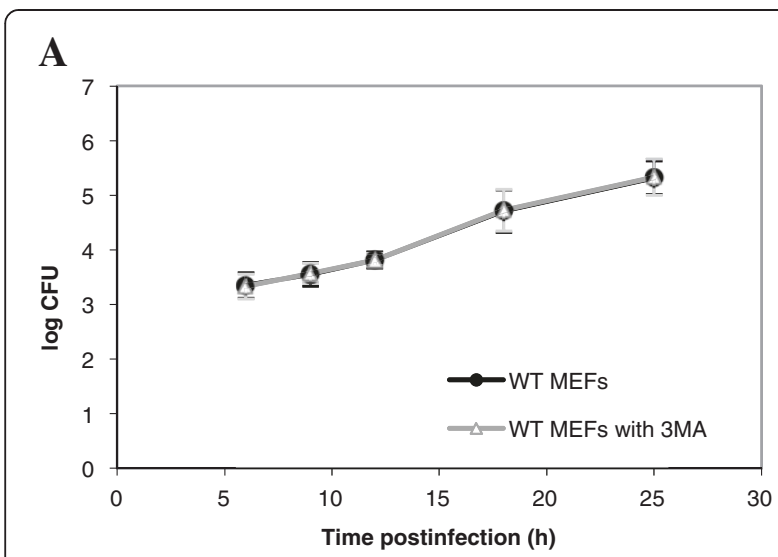

B

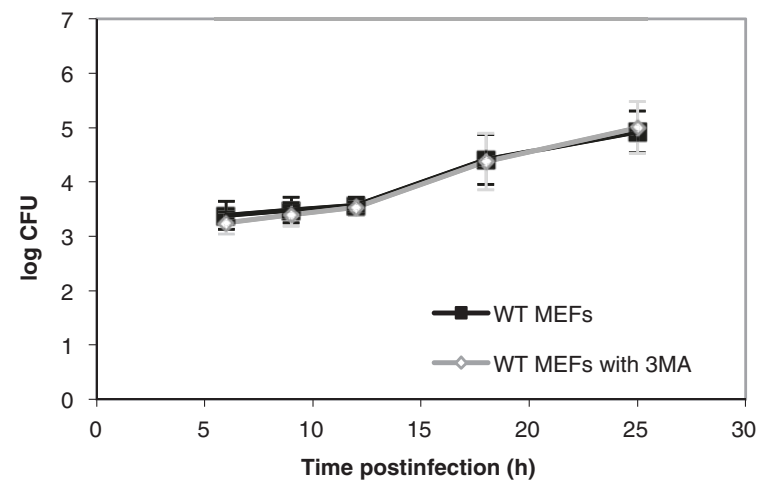

Figure 6 Impact of 3MA on the infection of WT MEFs with $B$. abortus S2308 (A) or with B. melitensis 16M (B). Results represent $\log$ CFUs (means \pm SD) measured at various times postinfection in at least three independent experiments made in triplicates.

cell, which takes into account living bacteria but also dead bacteria and bacteria that are no longer able to replicate, indicates that for $B$. abortus, there is no difference between the two cell lines even at short times postinfection (Figure 3A) whereas for B. melitensis, there is a significant increase in the Atg $5^{-1-}$ MEFs at $9,18 \mathrm{~h}$ and 24 h. p.i., as compared to WT MEFs (Figure 3B). Therefore, for B. abortus, the higher CFUs in Atg $5^{-1-}$ MEFs vs WT MEFs could be explained by an increase in the percentage of infected cells among the cell population or by a higher survival rate during the early times after infection rather than by a higher replication rate. In contrast, for $B$. melitensis, the increase in the $\log \mathrm{CFU}$ in Atg5-deficient cells could also result from a slight increase in the replication rate.

Next, our data reveal that there is no conversion of LC3-I to LC3-II in WT MEFs upon Brucella invasion and that neither B. abortus nor B. melitensis is detected in autophagic compartments stained with GFP-LC3, even under starvation conditions. This is consistent with the results of Starr et al. [12], which also showed that the siRNA-mediated silencing of LC3B in HeLa cells did not impair the maturation of the $\mathrm{BCV}$ into a replicative niche in cells infected with $B$. abortus. In contrast, Guo et al. [22] proposed that B. melitensis infection induced autophagy because they observed an accumulation of GFP-LC3-positive autophagic vacuoles and a conversion of LC3-I to LC3-II in infected RAW264.7 macrophages, compared to control cells. Moreover, these authors showed that a treatment with the autophagy inhibitor 3MA attenuated the replication efficiency of B. melitensis. It is not clearly indicated how long they incubated cells with this compound but it has been demonstrated that under nutrient-rich conditions, a prolonged treatment (up to $9 \mathrm{~h}$ ) with 3MA could promote rather than inhibit the autophagy flux [24]. In contrast to Guo et al., [22], we did not observe a significant decrease in the CFU and in the number of Brucella per infected cells (except for B. melitensis at 24 h p.i.) in WT MEFs pretreated with 3MA. This discrepancy could be explained either by the incubation conditions or by a cell-type specificity. The subversion of the autophagic pathway by $B$. melitensis could occur in RAW264.7 macrophages but not in MEFs.

Given the multifactorial effects of 3MA on cell metabolism [25], cells derived from Atg5 KO mice represent a more reliable tool to study the role of autophagy in different biological situations [18]. Based on our results with $\operatorname{Atg} 5^{-1-}$ MEFs, it is obvious that $B$. melitensis $16 \mathrm{M}$ as well as B. abortus are able to replicate in cells deficient in the canonical macroautophagy pathway. However, we cannot rule out the involvement of autophagosomes formed by an Atg5 and Atg7-independent alternative macroautophagy. Indeed, it has been demonstrated that the incubation of $\operatorname{Atg} 5^{-/-}$MEF with etoposide, a proapoptotic molecule, induced autophagosome formation without conversion of LC3-I to LC3-II [26]. Likewise, Starr et al. [12] have shown that the conversion of rBCVs into $\mathrm{aBCV}$ that occurs at a very late stage after infection with $B$. abortus does not require several core autophagic proteins, of which Atg5 and LC3B [12]. These findings demonstrate that autophagic vacuoles can be formed in Atg5deficient cells. However, these alternative macroautophagy pathways, independent of Atg 5 and LC3, are inhibited by 3MA [12,26]. Thus, if Brucella subverts an alternative macroautophagy pathway to reach its replicative niche in mouse embryonic fibroblasts, it should proceed by another mechanism because in our conditions of incubation, the replication efficiency is not impaired in WT MEFs treated with 3MA.

Finally, it has been demonstrated that the intracellular trafficking of $B$. abortus and B. melitensis could be different in some human trophoblastic cell lines [27]. Therefore, it could be interesting to study the involvement of the conventional and the alternative macroautophagy pathways in other cell types, such as trophoblasts and peritoneal or bone marrow-derived macrophages. 


\section{Conclusion}

Collectively, our data indicate on one hand that cell invasion with $B$. abortus and $B$. melitensis does not induce macroautophagy in WT MEFs and on the other hand, that both Brucella strains can replicate in Atg5-deficient MEFs.

\section{Methods}

\section{Bacteria strains}

Brucella abortus S2308 and Brucella melitensis $16 \mathrm{M}$ are $\mathrm{CO}_{2}$-independent virulent smooth strains. BrucellamCherry strains constitutively express the fluorescent mCherry protein due to the intregration of a plasmid containing the coding sequence of mCherry and a kanamycin resistance marker [28]. Before each infection, bacteria stored at $-80^{\circ} \mathrm{C}$ were plated onto 2YT Agar (1.6\% bacto-peptone, $1 \%$ yeast extract, $0.5 \% \mathrm{NaCl}$ and $1.3 \%$ Agar) Petri dishes. For Brucella-mCherry, kanamycin $(10 \mu \mathrm{g} / \mathrm{mL})$ was added in this culture medium to maintain selection. After approximately 72 hours of incubation at $37^{\circ} \mathrm{C}$, a dozen or so isolated colonies were taken and cultured overnight at $37^{\circ} \mathrm{C}$ under agitation in $5 \mathrm{~mL}$ of 2 YT liquid medium ( $1 \%$ tryptone, $0.6 \%$ bacto-peptone, $1 \%$ yeast extract and $0.5 \% \mathrm{NaCl}$ ) without antibiotics.

\section{Host cells}

We used mouse embryonic fibroblasts from wild type (WT MEFs) and from Atg5 knockout mice (Atg $5^{-1-} \mathrm{MEFs}$ ) [29] available at the Riken BRC Cell Bank. Cells were cultured in Dulbecco's modified Eagle medium (DMEM, Lonza) supplemented with $10 \% \mathrm{vol} / \mathrm{vol}$ fetal calf serum (FCS, Sigma). After counting in a Burker chamber, MEFs were seeded at a density of 50,000 cells/well in 12-well plates containing coverslips for the microscopy experiments and in 24-well plates in triplicates for the counting of CFUs. After seeding, cells were incubated overnight at $37^{\circ} \mathrm{C}$ under $5 \%$ of $\mathrm{CO}_{2}$ before bacteria inoculation. When indicated, $10 \mathrm{mM}$ 3-methyladenine (Sigma, directly prepared in DMEM medium) was added to the cell monolayers to inhibit autophagy prior to infection. To investigate the presence of Brucella in LC3B-positive autophagosomes, we established stable clones of MEFs expressing GFP-LC3 WT (plasmid pEX-GFP-hLC3WT, Addgene). Starvationinduced autophagy was obtained by a 2 h-incubation in EBSS medium (Earle's Balanced Salt solution) after three washes with PBS to remove serum.

\section{Cell infection with Brucella}

Growth of bacteria was assessed by measuring the optical density (OD) at a wavelength of $600 \mathrm{~nm}$ considering that an $\mathrm{OD}=1$ corresponds to $1 \times 10^{9}$ bacteria $/ \mathrm{mL}$. Then, bacteria were sedimented by centrifugation at $900 \mathrm{~g}$ for $10 \mathrm{~min}$ to discard 2YT medium and resuspended in the same volume of DMEM + 10\% FCS. After dilution of the bacterial suspension in an appropriate volume of DMEM + FCS to get an MOI (multiplicity of infection) of 300 , the culture medium present in 12-well plates containing MEFs was withdrawn and replaced by the bacterial suspension. The Petri dishes were centrifuged for $10 \mathrm{~min}$ at $400 \mathrm{~g}$ at $4{ }^{\circ} \mathrm{C}$ to favour the adhesion of bacteria to the cell surface and then placed in a $5 \% \mathrm{CO}_{2}$ incubator at $37^{\circ} \mathrm{C}$ (this is the time zero postinfection). The passage from $4^{\circ} \mathrm{C}$ to $37^{\circ} \mathrm{C}$ aims at synchronizing the entry of bacteria into the cells. After one hour of infection, wells were washed thrice with sterile phosphate-buffered saline (PBS, $136.9 \mathrm{mM} \mathrm{NaCl}, 2.7 \mathrm{mM} \mathrm{KCl}, 10.1 \mathrm{mM} \mathrm{Na}_{2} \mathrm{HPO}_{4}$ and $1.8 \mathrm{mM} \mathrm{KH}_{2} \mathrm{PO}_{4}$ ) and further incubated for one hour with DMEM + FCS containing $50 \mu \mathrm{g}$ of gentamicin per $\mathrm{mL}$ to kill extracellular bacteria. Afterwards, the medium was changed and replaced by the medium containing $10 \mu \mathrm{g}$ of gentamicin per $\mathrm{mL}$ until the end of the postinfection period [28].

For the counting of viable intracellular bacteria using colony forming units (CFUs), after infection with Brucella, cells were washed thrice with PBS then lysed for $10 \mathrm{~min}$ at room temperature in $800 \mu \mathrm{l}$ of PBS containing $0.1 \%$ Triton X-100 under manual agitation. Lysates were diluted from 10 to 1,000 times in PBS and plated on Petri dishes containing 2YT Agar. Petri dishes were incubated for three to four days at $37^{\circ} \mathrm{C}$ before the counting of colony forming units.

\section{Fluorescence microscopy}

To count the number of Brucella per infected cell, we infected MEFs with Brucella-mCherry. At various time points p.i., cells were washed twice with filtered dPBS (PBS supplemented with $0.88 \mathrm{mM} \mathrm{CaCl} 2$ and $0.5 \mathrm{mM} \mathrm{MgCl} 2$ ), fixed for $20 \mathrm{~min}$ at room temperature in $4 \%$ paraformaldehyde in cold PBS, then washed thrice with dPBS. Nuclei were stained with 4'-6-diamidino-2-phenylindole (DAPI) prepared in PBS containing 0.1\% Triton X-100 and washed three times with PBS. Coverslips were mounted in Mowiol on glass plates. Fluorescence was observed using a Nikon i80 fluorescence microscope. In an attempt to detect Brucella in compartments stained with LC3, we infected cells expressing GFP-LC3 with B. abortus S2308 or with B. melitensis $16 \mathrm{M}$ that do not express mCherry. After fixation, membrane permeabilisation with Triton X-100 $(0.1 \%$ in $\mathrm{DPBS}$ ) and blocking of unspecific sites with bovine serum albumine ( $2 \%$ in $\mathrm{dPBS})$, bacteria were detected with a monoclonal antibody raised against the lipopolysaccharides of Brucella (A76-12G12) [30] and a goat anti-mouse Texas Red-conjugated secondary antibody. Fluorescence was observed using a Leica TCD confocal fluorescence microscope.

\section{Western blotting}

MEFs were washed three times with PBS and then incubated for $10 \mathrm{~min}$ in cold lysis buffer $(10 \mathrm{mM}$ Tris- $\mathrm{HCl}$ 
pH 7.4, $150 \mathrm{mM} \mathrm{NaCl}, 0.5 \%$ Triton X-100 and a proteaseinhibitor cocktail (Roche)). After $10 \mathrm{~min}$ of rotation on a wheel, cell lysates were centrifuged for $15 \mathrm{~min}$ at 13,000 $\mathrm{RPM}$ at $4^{\circ} \mathrm{C}$ to sediment cell debris. Protein concentration of these clear lysates was determined using the BCA (Bicinchoninic acid) protein assay (Pierce). Fifteen micrograms of proteins were separated by SDS-PAGE $12 \%$ and then, transferred onto polyvinyl difluoride (PVDF) membranes. Membranes were blocked for $1 \mathrm{~h}$ in PBS containing $0.1 \%$ Tween 20 and $2 \%$ of blocking agent (GE Healthcare), then incubated for $2 \mathrm{~h}$ with a primary monoclonal antiLC3B antibody (NanoTools, Germany) and a secondary anti-mouse antibody conjugated to horseradish peroxidase (HRP). The activity of HRP was revealed by enhanced chemiluminescence (Perkin-Elmer).

\section{Statistical analysis}

Error bars indicate standard deviation (SD) or standard error of the mean (SEM) as indicated in the legend. Statistical significance was determined using SigmaPlot 11 software. Whenever possible, we have performed unpaired Student's $t$-tests. When the normality test (Shapiro-Wilk) or the equal variance test failed, we carried out a Mann-Whitney rank sum test. A two-way ANOVA followed by a pairwise multiple comparison procedure (Holm-Sidak method) was also carried out. Statistical significant differences were accepted for $p<0.05$.

\section{Ethics statement}

No live animal was used in this work.

\section{Additional file}

\section{Additional file 1: GFP-LC3 labelling in WT MEFs infected or not} with B. abortus or B. melitensis. WT MEFs stably expressing GFP-LC3 were maintained under normal conditions (left) or under starved conditions (right). NI, BA and BM correspond to non infected cells, cells infected with $B$. abortus and cells infected with $B$. melitensis, respectively. MEFs were fixed at $10 \mathrm{~h}$ p.i. Bacteria were detected with a monoclonal anti-LPS antibody and an anti-mouse IgG Texas Red-conjugated secondary antibody. Nuclei were stained with DAPI. Cells were observed by confocal fluorescence microscopy.

\section{Abbreviations}

MEFs: Mouse embryonic fibroblasts; MOI: Multiplicity of infection; CFU: Colony forming units; WT: Wild-type; 3MA: 3-methyladenine.

\section{Competing interests}

The authors declare that they have no competing interests.

\section{Authors' contributions}

$I H, M J, X D B, J J L$ conceived the study. IH and EG carried out the experiments. $\mathrm{IH}$ wrote the manuscript and all the authors read and approved the final manuscript.

\section{Acknowledgments}

We acknowledge Dr. Noboru Mizushima (Tokyo Medical and Dental University) for providing WT and Atg $5^{-/-}$MEFs. This work was supported by the Actions de Recherches Concertées-Communauté Française de Belgique (Grant number Convention No8/13-015) and the University of Namur. We thank Thierry Arnould and Martine Raes (URBC, University of Namur) for fruitful discussions and access to the confocal microscopy.

\section{Author details}

${ }^{1}$ Research Unit in Molecular Physiology (URPhyM), NAmur Research Institute for LIfe Sciences (NARILIS), University of Namur, Namur, Belgium. ${ }^{2}$ Research Unit in Biology of Microorganisms (URBM), NAmur Research Institute for LIfe Sciences (NARILIS), University of Namur, Namur, Belgium. ${ }^{3}$ Present address: Faculty of Veterinary Medicine-Department of infectious and parasitic diseases, Laboratory of Immunology and Vaccinology, University of Liège, Liège, Belgium.

Received: 17 April 2014 Accepted: 13 August 2014

Published: 2 September 2014

\section{References}

1. Cemma M, Brumell JH: Interactions of pathogenic bacteria with autophagy systems. Curr Biol 2012, 22(13):R540-R545.

2. Vergne I, Fratti RA, Hill PJ, Chua J, Belisle J, Deretic V: Mycobacterium tuberculosis phagosome maturation arrest: mycobacterial phosphatidylinositol analog phosphatidylinositol mannoside stimulates early endosomal fusion. Mol Biol Cell 2004, 15(2):751-760.

3. Amer AO, Swanson MS: Autophagy is an immediate macrophage response to Legionella pneumophila. Cell Microbiol 2005, 7(6):765-778.

4. Romano PS, Gutierrez MG, Beron W, Rabinovitch M, Colombo MI: The autophagic pathway is actively modulated by phase II Coxiella burnetii to efficiently replicate in the host cell. Cell Microbiol 2007, 9(4):891-909.

5. Schnaith A, Kashkar H, Leggio SA, Addicks K, Kronke M, Krut O: Staphylococcus aureus subvert autophagy for induction of caspase-independent host cell death. J Biol Chem 2007, 282(4):2695-2706.

6. Starr T, Ng TW, Wehrly TD, Knodler LA, Celli J: Brucella intracellular replication requires trafficking through the late endosomal/lysosomal compartment. Traffic 2008, 9(5):678-694.

7. Arellano-Reynoso B, Lapaque N, Salcedo S, Briones G, Ciocchini AE, Ugalde R Moreno E, Moriyon I, Gorvel JP: Cyclic beta-1,2-glucan is a Brucella virulence factor required for intracellular survival. Nat Immunol 2005, 6(6):618-625.

8. Celli J, de Chastellier C, Franchini DM, Pizarro-Cerda J, Moreno E, Gorvel JP. Brucella evades macrophage killing via VirB-dependent sustained interactions with the endoplasmic reticulum. J Exp Med 2003, 198(4):545-556.

9. Pizarro-Cerda J, Moreno E, Gorvel JP: Invasion and intracellular trafficking of Brucella abortus in nonphagocytic cells. Microbes Infect 2000, 2(7):829-835.

10. Celli J: Surviving inside a macrophage: the many ways of Brucella. Res Microbiol 2006, 157(2):93-98.

11. Pizarro-Cerda J, Meresse S, Parton RG, van der Goot G, Sola-Landa A, Lopez-Goni I, Moreno E, Gorvel JP: Brucella abortus transits through the autophagic pathway and replicates in the endoplasmic reticulum of nonprofessional phagocytes. Infect Immun 1998, 66(12):5711-5724.

12. Starr T, Child R, Wehrly TD, Hansen B, Hwang S, Lopez-Otin C, Virgin HW, Celli J: Selective subversion of autophagy complexes facilitates completion of the Brucella intracellular cycle. Cell Host Microbe 2012, 11(1):33-45.

13. Pizarro-Cerda J, Moreno E, Sanguedolce V, Mege JL, Gorvel JP: Virulent Brucella abortus prevents lysosome fusion and is distributed within autophagosome-like compartments. Infect Immun 1998, 66(5):2387-2392.

14. Lamb CA, Yoshimori T, Tooze SA: The autophagosome: origins unknown, biogenesis complex. Nat Rev Mol Cell Biol 2013, 14(12):759-774.

15. Mizushima N, Yoshimori T, Ohsumi Y: The role of Atg proteins in autophagosome formation. Annu Rev Cell Dev Biol 2011, 27:107-132.

16. Hanada T, Noda NN, Satomi Y, Ichimura Y, Fujioka Y, Takao T, Inagaki F, Ohsumi Y: The Atg12-Atg5 conjugate has a novel E3-like activity for protein lipidation in autophagy. J Biol Chem 2007, 282(52):37298-37302

17. Mizushima N, Kuma A, Kobayashi Y, Yamamoto A, Matsubae M, Takao T, Natsume T, Ohsumi Y, Yoshimori T: Mouse Apg16L, a novel WD-repeat protein, targets to the autophagic isolation membrane with the Apg12-Apg5 conjugate. J Cell Sci 2003, 116(Pt 9):1679-1688.

18. Mizushima N, Yamamoto A, Hatano M, Kobayashi Y, Kabeya Y, Suzuki K, Tokuhisa T, Ohsumi Y, Yoshimori T: Dissection of autophagosome formation using Apg5-deficient mouse embryonic stem cells. J Cell Biol 2001, 152(4):657-668. 
19. Kabeya Y, Mizushima N, Ueno T, Yamamoto A, Kirisako T, Noda T, Kominami E, Ohsumi Y, Yoshimori T: LC3, a mammalian homologue of yeast Apg8p, is localized in autophagosome membranes after processing. EMBO J 2000, 19(21):5720-5728.

20. Tanida I, Sou YS, Ezaki J, Minematsu-lkeguchi N, Ueno T, Kominami E: HsAtg4B/HsApg4B/autophagin-1 cleaves the carboxyl termini of three human Atg8 homologues and delipidates microtubule-associated protein light chain 3- and GABAA receptor-associated protein-phospholipid conjugates. J Biol Chem 2004, 279(35):36268-36276.

21. Tanida I, Ueno T, Kominami E: Human light chain 3/MAP1LC3B is cleaved at its carboxyl-terminal Met121 to expose Gly120 for lipidation and targeting to autophagosomal membranes. J Biol Chem 2004, 279(46):47704-47710.

22. Guo F, Zhang H, Chen C, Hu S, Wang Y, Qiao J, Ren Y, Zhang K, Wang Y, Du G: Autophagy favors Brucella melitensis survival in infected macrophages. Cell Mol Biol Lett 2012, 17(2):249-257.

23. Seglen PO, Gordon PB: 3-Methyladenine: specific inhibitor of autophagic/ lysosomal protein degradation in isolated rat hepatocytes. Proc Natl Acad Sci U S A 1982, 79(6):1889-1892.

24. Wu YT, Tan HL, Shui G, Bauvy C, Huang Q, Wenk MR, Ong CN, Codogno P, Shen HM: Dual role of 3-methyladenine in modulation of autophagy via different temporal patterns of inhibition on class I and III phosphoinositide 3-kinase. J Biol Chem 2010, 285(14):10850-10861.

25. Caro LH, Plomp PJ, Wolvetang EJ, Kerkhof C, Meijer AJ: 3-Methyladenine, an inhibitor of autophagy, has multiple effects on metabolism. Eur J Biochem 1988, 175(2):325-329.

26. Nishida Y, Arakawa S, Fujitani K, Yamaguchi H, Mizuta T, Kanaseki T, Komatsu M, Otsu K, Tsujimoto Y, Shimizu S: Discovery of Atg5/Atg7-independent alternative macroautophagy. Nature 2009, 461(7264):654-658.

27. Salcedo SP, Chevrier N, Santos Lacerda TL, Ben Amara A, Gerart S, Gorvel VA, de Chastellier C, Blasco JM, Mege JL, Gorvel JP: Pathogenic Brucellae replicate in human trophoblasts. J Infect Dis 2013, 207(7):1075-1083.

28. de Barsy M, Jamet A, Filopon D, Nicolas C, Laloux G, Rual JF, Muller A, Twizere JC, Nkengfac B, Vandenhaute J, Hill DE, Salcedo SP, Gorvel JP, Letesson JJ, De Bolle X: Identification of a Brucella spp. secreted effector specifically interacting with human small GTPase Rab2. Cell Microbiol 2011, 13(7):1044-1058

29. Kuma A, Hatano M, Matsui M, Yamamoto A, Nakaya H, Yoshimori T, Ohsumi Y, Tokuhisa T, Mizushima N: The role of autophagy during the early neonatal starvation period. Nature 2004, 432(7020):1032-1036.

30. Cloeckaert A, Zygmunt MS, Dubray G, Limet JN: Characterization of O-polysaccharide specific monoclonal antibodies derived from mice infected with the rough Brucella melitensis strain B115. J Gen Microbiol 1993, 139(7):1551-1556.

doi:10.1186/s12866-014-0223-5

Cite this article as: Hamer et al:: Replication of Brucella abortus and Brucella melitensis in fibroblasts does not require Atg5-dependent macroautophagy. BMC Microbiology 2014 14:223.

\section{Submit your next manuscript to BioMed Central and take full advantage of:}

- Convenient online submission

- Thorough peer review

- No space constraints or color figure charges

- Immediate publication on acceptance

- Inclusion in PubMed, CAS, Scopus and Google Scholar

- Research which is freely available for redistribution

Submit your manuscript at www.biomedcentral.com/submit
Ciomed Central 